\title{
Gastroduodenal perforation in preterm babies treated with dexamethasone for bronchopulmonary dysplasia
}

\author{
P C Ng, K G Brownlee, P R F Dear
}

\begin{abstract}
Three cases of gastroduodenal perforation and one case of ulceration and extreme thinning of the gastric wall occurred in preterm babies treated with dexamethasone for bronchopulmonary dysplasia. This complication of treatment with steroids has not to our knowledge previously been described in preterm infants. Urgent abdominal paracentesis was an essential part of the resuscitation in these cases, and this potentially serious side effect should be considered in all babies treated with steroids.
\end{abstract}

Treatment with corticosteroids often produces adverse side effects in both adults and children. Whether or not preterm infants differ in susceptibility to some or all of these adverse effects is not yet known, as they have not yet been studied extensively; it is, therefore, important to report all adverse side effects of corticosteroids in this age group so that the risk:benefit ratio can be evaluated and potential adverse effects anticipated.

In this report we describe two cases of gastric perforation, one case of duodenal perforation, and one case of extreme thinning of the stomach wall among preterm babies being treated with dexamethasone for bronchopulmonary dysplasia in the neonatal unit at St James's University Hospital, Leeds, and Huddersfield Royal Infirmary, over a four year period.

\section{Case reports}

CASE 1

A male second twin who was born at 30 weeks' gestation weighing $1469 \mathrm{~g}$ developed bronchopulmonary dysplasia after severe respiratory distress syndrome complicated by air leaks. On day 18 he developed necrotising enterocolitis, which responded to medical treatment.

During the ensuing weeks several attempts at extubation were unsuccessful because of chronic lung disease, and a three week course of dexamethasone was started on day 48 . The initial dose was $0.6 \mathrm{mg} / \mathrm{kg} /$ day, reducing to $0.3 \mathrm{mg} /$ $\mathrm{kg} /$ day in the second week, and to $0.15 \mathrm{mg} / \mathrm{kg} /$ day in the third week. He was successfully weaned off the ventilator on day 51 and enteral feeding was started the next day. Two days later he had bloodstained nasogastric aspirates, but clotting screen and platelet count were normal. No bleeding sites or bruises were observed elsewhere. He was given cimetidine $20 \mathrm{mg} / \mathrm{kg} /$ day for three days and the episode of bleeding stopped spontaneously within 48 hours. On day 69 (the final day of his three week course of dexamethasone) and while receiving alternate bottle and nasogastric feeds of expressed breast milk, he suddenly collapsed with episodes of serious apnoea and bradycardia. He became hypotensive and cyanosed. His abdomen became grossly distended within an hour and an abdominal $x$ ray film showed pneumoperitoneum (figure) but no evidence of necrotising enterocolitis. Immediate resuscitative measures included positive pressure ventilation, and giving atropine, sodium bicarbonate, and a plasma expander. An abdominal drain was inserted, and $60 \mathrm{ml}$ of free gas released from the abdomen, which facilitated mechanical ventilation.

Laparotomy was done within three hours and a perforation was found on the anterior aspect of the lesser curvature of the stomach. There was negligible soiling of the peritoneal cavity and the intestines were healthy. The lesion was oversewn, the baby was weaned off the ventilator within 72 hours, and enteral feeding was restarted. He made an uneventful recovery and was discharged home within a month.

CASE 2

This female infant was the third of triplets born

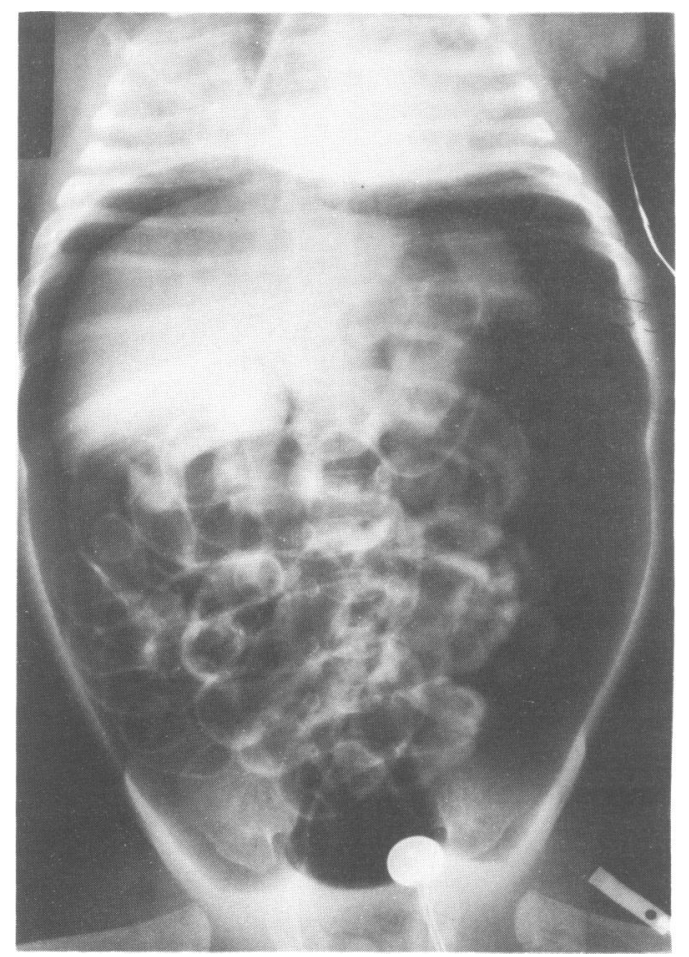

Case 1: abdominal radiograph (supine) showing tension pneumoperitoneum compressing the thorax. 
at 28 weeks' gestation weighing $895 \mathrm{~g}$. Her neonatal problems included severe respiratory distress syndrome complicated by early pulmonary interstitital emphysema, multiple pneumothoraces, and rapid progression to bronchopulmonary dysplasia. A similar three week course of decreasing doses of dexamethasone was started on day 7. Little progress was made in weaning her from intermittent positive pressure ventilation (IPPV), however, so on day 47 a second course of dexamethasone was started. The dose was similar to the previous one but continued after three weeks with a dose on alternate days of $0 \cdot 15 \mathrm{mg} / \mathrm{kg} /$ day. During this period she was successfully weaned from IPPV into $50 \%$ headbox oxygen. On day 90 , while taking full nasogastric milk feeds, she passed melaena stools, but this episode resolved spontaneously without any treatment after 48 hours. On day 95 she suddenly collapsed with gross abdominal distension, shock, respiratory arrest, and bradycardia. Fresh blood was aspirated from an indwelling nasogastric tube and abdominal $x$ ray film confirmed the presence of free intraperitoneal gas. Effective ventilation was only achieved after aspiration of $50 \mathrm{ml}$ of free gas from the abdomen.

At laparotomy five hours later a longitudinal perforation was found on the posterior wall of the stomach and the lesion was oversewn. The stomach lining was atrophic and thin. Omeprazole was given immediately after the operation to reduce the output of gastric acid. No further gastrointestinal bleeding occurred and enteral feeding was restarted 10 days after the operation. During her convalescence it became clear that the baby had sustained hypoxic/ischaemic brain injury at the time of the perforation. She died six weeks later of severe bronchopulmonary dysplasia and respiratory failure.

CASE 3

A female infant was born at 27 weeks' gestation weighing $980 \mathrm{~g}$. She developed bronchopulmonary dysplasia after moderate respiratory distress syndrome which was complicated by a left sided pneumothorax. Nasogastric feeding was started on day 2 . She was extubated on day 14 and required $28 \%$ oxygen. At 3 weeks her oxygen requirements started to increase and by 6 weeks she required $50 \%$ oxygen. A chest $x$ ray film confirmed that her bronchopulmonary dysplasia was worsening and a course of decreasing doses of dexamethasone was started at 6 weeks, at an initial dose of $0.6 \mathrm{mg} / \mathrm{kg}$. Within three days her oxygen requirements had fallen to $25 \%$. Nine days after the dexamethasone had been started she became acutely ill with circulatory collapse, and required volume expanders and cardiopulmonary resuscitation. There was pronounced abdominal distension and an abdominal radiograph showed pneumoperitoneum but no evidence of necrotising enterocolitis.

She was transferred to the regional neonatal surgical unit where at laparotomy a duodenal perforation was confirmed. There was minimal peritoneal contamination and the remaining bowel looked healthy. The lesion was oversewn and the baby was weaned off ventilation after six days. After operation she was given a four week course of cimetidine. She made an uneventful recovery and was discharged home at 3 months of age. She was reviewed at the age of 5 months, and despite a follow up cranial ultrasound scan that showed bilateral periventricular leucomalacia, was progressing normally.

CASE 4

A female infant was born at 24 weeks' gestation weighing $610 \mathrm{~g}$. She had respiratory distress syndrome and required IPPV from birth. Her course was complicated by hypotension and she required inotropic drugs. She had a persistent ductus arteriosus, which was treated with indomethacin, congenital hypothyroidism that required replacement therapy, and bilateral grade II intraventricular haemorrhages. She developed severe bronchopulmonary dysplasia and was treated with intermittent courses of dexamethasone $(0.6 \mathrm{mg} / \mathrm{kg} / \mathrm{day})$ in an attempt to wean her off the ventilator. The first two courses were interrupted after only a few days because infection was suspected. Dexamethasone was given for a total of 21 days, of which 18 were during the last month of life.

Despite treatment, her pulmonary function continued to deteriorate and she died on day 64 while still receiving steroids. At necropsy two areas of apparently active ulceration were found near the top of the greater curvature of the stomach on the anterior surface, where the wall was extremely thin though there was no obvious perforation.

\section{Discussion}

A Medline computer search of papers written in English from 1970-89 inclusive failed to find any reports of gastrointestinal perforation that could have been attributed to the use of corticosteroids in newborns, though it is well documented in adults. ${ }^{1-3}$ Proposed mechanisms include: inhibition of the mucosal cell generative mechanism and the rate of gastric cell turnover ${ }^{13}$; decreased synthesis of antral mucus $^{3}$; increased susceptibility of intestinal wall to infection ${ }^{1}$; thinning of the intestinal wall secondary to depletion of lymphoid patches ${ }^{1}$; or synergism with other risk factors such as stress induced ulcer, faecal impaction, or drugs such as theophylline or caffeine that lower the threshold to perforation. ${ }^{4-6}$ Interestingly, there is no reported correlation between the risk of perforation and the dose ${ }^{1}$ or the route of administration. ${ }^{3}$

Stress induced gastric ulceration is an improbable cause of perforation in our cases as none of the babies was receiving IPPV and all were convalescing in the low dependency area of the unit. All were receiving caffeine or aminophylline, but so were most of our very low birthweight babies. Furthermore, a review of admissions to St James's University Hospital during the last 10 years failed to show any other cases of gastroduodenal perforation.

In contrast to many of the reported cases, the anti-inflammatory effect of corticosteroids did 
not mask the evolution of abdominal signs in the babies. ${ }^{1-3}$ The sequence of events was alarmingly rapid and all three babies became moribund within half an hour of the onset of signs. Abdominal distension was so tense that it prevented diaphragmatic movement, and paracentesis with a butterfly needle was life saving. Positive pressure ventilation and vigorous plasma expansion were essential supportive measures during the initial resuscitation period.

The incidence of gastric perforation in preterm babies receiving dexamethasone in our neonatal unit seems to be in the region of $2 \%$ to $3 \%$, in keeping with the finding in adults. ${ }^{1}$ The necropsy findings in case 4, however, suggest that there might be cases of active ulceration that go undetected. Two of the babies who had perforations had had an episode of gastrointestinal bleeding that had not been accounted for, although this had stopped days before the perforation occurred.

We suggest that unexplained melaena or bloodstained gastric aspirates in a baby receiving steroids should be regarded as a sign of impending perforation and possibly as an indication to stop steroids. Whether the risk of this potentially life threatening side effect can be reduced by giving antacids, $\mathrm{H}_{2}$ receptor blockers, or one of the new generation of hydrogen ion ATPase inhibitors such as omeprazole, needs to be investigated.

We thank Dr M Miller for allowing us to report a case under his care.

1 Fadul CE, Lemann W, Thaler HT, Posner JB. Perforation of the gastrointestinal tract in patients receiving steroids for the gastrointestinal tract in patients receiving steroin

2 ReMine SG, Mcllrath DC. Bowel perforation in steroidtreated patients. Ann Surg 1980;192:581-6.
tolling DC. Bowel perforation

3 Dayton MT, Kleckner SC, Brown DK. Peptic ulcer perforation associated with steroid use. Arch Surg 1987;122 376-80.

4 Guss C, Schneider AT, Chiaramonte LT. Perforated gastric ulcer in an asthmatic treated with theophylline and steroids. Case report and literature review. Ann Allergy $1986 ; 56: 237-40$.

5 Debas HT, Cohen MM, Holybitsky IB, et al. Caffeine stimulated gastric acid and pepsin secretion; dose response studies. Scand $\mathcal{f}$ Gastroenterol 1971;6:453-7.

6 Roth JA, Ivy AC. Comment: caffeine and peptic ulcer. Gastroenterology 1946;7:576-82. 